\title{
How to choose the right planning horizon? Using multi- objective optimization to support urban planning
}

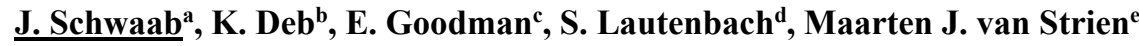 \\ and Adrienne Grêt-Regamey ${ }^{\mathrm{f}}$ \\ a Institute for Spatial and Landscape Planning, ETH Zürich, Zürich, Switzerland \\ ${ }^{b}$ Department of Electrical and Computer Engineering, Michigan State University, East Lansing, USA \\ ${ }^{c}$ BEACON-NSF Center for the Study of Evolution in Action, Michigan State University, East Lansing, USA \\ ${ }^{d}$ Department of Urban Planning and Real Estate Management, Institute of Geodesy and Geoinformation- \\ $I G G$, University Bonn, Bonn, Germany \\ e Institute for Spatial and Landscape Planning, ETH Zürich, Zürich, Switzerland \\ ${ }^{f}$ Institute for Spatial and Landscape Planning, ETH Zürich, Zürich, Switzerland \\ Email:jonasschwaab@ethz.ch
}

\begin{abstract}
To be useful to urban planners, urban models need to deliver plausible futures. However, the complexity of urban processes and related uncertainties make it difficult not only for urban planners, but also for urban models, to deliver plausible futures. Such uncertainties may for example involve population growth and the preferences of different stakeholders, which may change over time. In addition, urban planning outcomes are multi-dimensional and usually there are many trade-offs involved. To account for uncertainties in a multi-dimensional context we propose a modelling approach based on multi-objective optimization. We applied this approach in an artificial experiment and to a city in Switzerland accounting for two objectives. These were to preserve high-quality agricultural soils and to guarantee compact city development. As the most productive soils are often located close to existing urban areas, these two objectives are strongly conflicting and can cause a large trade-off. Our methodological approach involved two steps. First, in an artificial experiment, we demonstrated that uncertainties related to population growth or choosing a wrong planning horizon can prevent planners from reaching optimal solutions. We then tested the results from the artificial experiment for a real-world problem, which was the city of Uster in Switzerland. As the real-world problem was very complex, we used a metaheuristic for solving the multi-objective optimization problem of allocating new urban areas in all possible ways that would protect the most fertile soils and guarantee the compactness of the urban patterns. The used metaheuristic was the genetic algorithm NSGA-II. In order to account for the spatial nature of the optimization problem, we used a modified version of the original algorithm. After this, we ran the modified NSGA-II many times in order to create several Pareto Fronts for different planning horizons or if including uncertainties in population growth. We show that despite uncertainties and even when choosing a short planning horizon we may still be able to reach optimal urban patterns in a distant future, which is surprising as we are dealing with a highly non-linear combinatorial problem. In conclusion, the methodology we developed could help urban and spatial planners to identify the right planning horizon for a large variety of urban and spatial planning problems. In addition, the results from the multi-objective optimization can be used to improve interactive decision-making processes. By not only presenting a limited number of scenarios, but a whole range of different Pareto-optimal trade-off solutions to the decision-makers, they may be able to choose an efficient solution that fits their preferences very well.
\end{abstract}

Keywords: Multi-objective optimization, urban planning, peri-urban agriculture, preserving soil quality 


\section{INTRODUCTION}

Globally large amounts of agriculture land are converted into urban areas, which has far-reaching impacts on ecosystem services and the soil's capacity for agricultural production (FAO and ITPS, 2015). To reduce these impacts, it is possible either to reduce the amount of open land that is converted into urban or to find urban patterns that are less detrimental to the environment. If the amount of agricultural areas being converted into urban is reduced, this clearly diminishes the environmental impact. However, it may be much more difficult to identify urban patterns that best preserve good quality agricultural soils and at the same time account for economic objectives like reducing infrastructure costs.

Urban planners are facing the difficulty of designating agricultural land to the future development of urban sites - a process usually called zoning. Designing sustainable zoning plans is a wicked decision-making challenge because a variety of stakeholders and decision makers with conflicting values are involved (Artmann, 2015), deep uncertainties need to be considered (Walker et al., 2013) and even the formulation of the problem itself can be contested (Rittel and Webber, 1973). Because of these properties of wicked problems, Kwakkel et al. (2016) point out that decision making in wicked problem situations should be understood as an argumentative process.

To support urban planners and decision-makers in the argumentative process of finding sustainable solutions for urban growth, a large variety of quantitative tools and approaches has been developed. Malczewski (2015) divides these approaches into simulation and optimization approaches. He explains that simulation approaches may be characterized as descriptive and that optimization approaches are often considered to be normative. In short, normative approaches are concerned with the question "what ought to be", while descriptive models rather reflect decision-making agents and processes for answering the question "what if" or "what is".

A variety of studies has proposed to use multi-objective optimization in order to support decision-makers in urban planning (e.g. Haque and Asami, 2014, Caparros-Midwood et al., 2015, Schwaab et al., 2017). The aim of multi-objective optimization is to create many optimal trade-off solution, i.e., a set of Pareto optimal solutions called the Pareto Front, so that stakeholders and decision-makers can select one of them according to their preferences. Providing optimal trade-off solutions to stakeholders and decision-makers is a normative approach, which can be very helpful as it allows them to explore many potential solutions and balance their preferences well. However, a purely normative approach may not be sufficient in order to thoroughly support decision-makers in wicked problem situations where an argumentative process is essential. We argue that multi-objective optimization offers a large spectrum of methodological approaches and applications. Instead of only answering the question "what ought to be" it may as well be possible to answer the questions "what if" and in particular to show how we may deal with uncertainties related to the challenge of reaching sustainable urban growth.

In this study, we provide the reader with a novel approach on how to use multi-objective optimization. We demonstrate in which way multi-objective optimization may help us to deal with the problem of uncertain demands for new urban areas and the problem of choosing the right planning-horizon, when proposing optimal allocation of new urban zones. In order to show this, we formulate the bi-objective problem of maximizing compact urban development while minimizing the loss of good quality agricultural soils, which are two important aims of the Swiss planning legislation. In order to analyze whether short or long-term planning may be more useful considering uncertainties in the demand for new urban areas, we apply a stepwise optimization process, which we explain in detail.

\section{METHODS}

\subsection{Problem formulation}

Our goal was to optimize the allocation of new urban areas for a small artificial landscape and in the municipality of Uster, which is situated in the canton of Zürich in Switzerland. We focused on optimizing two objectives. One was to minimize the loss of agricultural soil quality. The other was to maximize compactness of urban areas. As a first constraint, urban areas were only allowed to be built on agricultural land and not on other areas (e.g. forests). Second, the amount (i.e. demand) of urban areas to be allocated was fixed.

The soil quality loss (SQL) was calculated as following (notation adapted from Stewart et al. (2004)): 


$$
S Q L(u)=\frac{1}{S} \sum_{r=1}^{R} \sum_{c=1}^{C} \sum_{k=1}^{K} s_{r c k} x_{r c k}
$$

where $\mathrm{u}$ denotes the specific land-use map expressed in terms of $\mathrm{R} \times \mathrm{C} \times \mathrm{K}$ binary variables $x_{r c k}$, such that $x_{r c k}=1$ if $u_{r c}=k$, and $x_{r c k}=0$ otherwise, $\mathrm{R}$ is the number of rows, $\mathrm{C}$ is the number of columns, $\mathrm{K}$ is the number of possible LULC categories (i.e., in this study: urban or agriculture), $s_{r c k}$ is the potential loss of soil quality and $\mathrm{S}$ is the sum of the currently available agricultural soil quality. For more details on how the soil quality loss has been calculated please refer to Schwaab et al. (2017).

We decided to calculate the Total Edge Length (TEL) as an indicator for compactness. The TEL is inversely related to compactness, meaning that a low TEL describes a compact pattern (McGarigal et al., 2012). The TEL was calculated as (notation adapted from Aerts et al. (2003):

$$
\operatorname{TEL}(u)=\sum_{r=1}^{R} \sum_{c=1}^{C} \sum_{k=1}^{K}\left(\begin{array}{c}
x_{r+1, c, k}+x_{r, c+1, k} \\
+x_{r-1, c, k}+x_{r, c-1, k}
\end{array}\right)
$$

where $u$ denotes the specific land-use map expressed in terms of $\mathrm{R} \times \mathrm{C} \times \mathrm{K}$ binary variables $x_{r c k}$. The variables $x_{r+1, c, k}, x_{r-1, c, k}, x_{r, c+1, k}$ and $x_{r, c-1, k}$ are binary variables describing the von Neumann neighbourhood of a centre cell being assigned to a specific land-use $\left(u_{r c}\right)$. If a neighbour and the centre are assigned to the same land-use (e.g. $u_{r+1, c}=u_{r c}$ ), the binary neighbourhood variable is zero (e.g., $x_{r+1, c, k}=0$ ). If the neighbour and the center are assigned to different land-uses (e.g, $u_{r+1, c} \neq u_{r c}$ ), the neighbourhood variable is one (e.g., $\left.x_{r+1, c, k}=1\right)$.

\subsection{Algorithm}

The aim of a multi-objective optimization algorithm is to find the so-called Pareto Front. The Pareto Front contains all Pareto optimal solutions. A solution is Pareto optimal if the value of one objective can only be improved in trade-off with the value of at least one other objective (Pareto, 1896). As we are using a stochastic optimization algorithm (more specifically an evolutionary algorithm) to solve the optimization problem, there is no guarantee that we are able to find the true Pareto Front. Thus, in this study we will mostly speak of the non-dominated front, which expresses the fact that the solutions obtained with the used algorithm may not be truly Pareto optimal, but they are not dominated by any other solution we evaluated.

To solve our multi-objective optimization problem, we used the elitist Non-dominated Sorting Genetic Algorithm (NSGA II, Deb et al., 2002). We modified the recombination and mutation operators to suit our specific optimization problem and implemented the algorithm based on the python framework "Distributed Evolutionary Algorithms in Python" (DEAP, Fortin et al., 2012).

Modifications of the recombination and mutation operators were mainly used to account for the spatial nature of the problem. For the recombination we split the parent solutions into two halves by a line through the center of the municipality with randomly varying angles, which is similar to an approach that has been recently applied for a spatial problem (Ryerkerk et al., 2012). The mutation process used in this genetic algorithm was basically a combination of uniform and biased mutation operators. More details on the modification of crossover and mutation operators can be found in Schwaab et al. (2017). To monitor the performance of the optimization process we calculated the hypervolume indicator at each iteration (Zitzler, 1999).

\subsection{Comparison of short- and long-term planning}

To simulate short- and long-term planning, we used identical genetic algorithms. However, we defined different demands for new urban areas and instead of running the algorithm one time in the long-term case, we applied a stepwise optimization procedure to simulate short-term planning. For both short- and long-term planning, the optimization started based on the land-use pattern in 2010. For the stepwise short-term planning process, we optimized the allocation of new building zones from 2010 until 2030. From the obtained nondominated front we selected one pattern, which we assumed to be the development path chosen by the decisionmakers (i.e., the preference according to the two objectives considered). This pattern was then used as the new starting pattern for the optimization from 2030 until 2050. For each solution (i.e. pattern) that we selected from the non-dominated front created for 2030, we created a new non-dominated front in 2050 (i.e. several 20302050 fronts). For the long-term planning process, we ran the genetic algorithm only one time and produced one font for the year 2050 (i.e. one 2010 - 2050 front) as there was only one starting pattern, which is the one from 
2010 based on the Swiss Area Statistics (Humbel, 2009). The demand for new urban areas between 2010 and 2050 was estimated based on population forecasts and data about the urban area per capita.

Both, the population forecast as well as the prediction of the future urban area per capita were highly uncertain. As urban planners will try to avoid oversized building zones they may choose a very conservative demand estimate. To account for this, we predicted the demand until 2050 using the lower $95 \%$ boundary of the confidence interval of the population forecast instead of the median forecast. The resulting demand was 174 ha of new urban areas instead of 212 ha. However, we would like to know if this more conservative demand estimate would prevent reaching optimal urban patterns if the demand turns out to be higher. Thus we also extended that run from the 174 ha solution by adding 38 ha of additional demand, to reach the demand of 212 ha, which was the demand if using the median population growth prediction until 2050. Note that this experiment was equivalent to the comparison of short- and long-term planning described in the previous paragraph. However, the time periods would be slightly different here. Using the lower demand (based on the lower $95 \%$ confidence interval boundary) in the optimization process would correspond to short-term planning from 2010 to approximately 2043 . From 2043 to 2050 the difference in the demand $(212-174=38$ ha) has to be added to reach the full demand through 2050.

\section{RESULTS AND DISCUSSION}

\subsection{Toy Experiment}

Short-term urban planning can result in non-optimal solutions in comparison to long-term planning (Figure 1). This can happen due to the non-linear combinatorial nature of the problem. As our simplified experiment showed, there are two ways of detecting whether short-term planning does result in non-optimal solutions when considering a longer time horizon. First, it is possible to analyze the non-dominated solutions resulting from short- and long-term planning in the objective space. If there is a gap between solutions on these fronts (Figure $1 \mathrm{~b}$ ), it is most likely that short-term planning (i.e., the optimization split into two smaller parts) is not able to produce optimal solutions in the long run. Second, if the non-dominated solutions reveal differences in the decision space, this will most likely also mean that the short-term planning has resulted in non-optimal solutions. This would mean that the final urban patterns look different (as was the case in our example in 2050, Figure $1 \mathrm{a})$.

a)

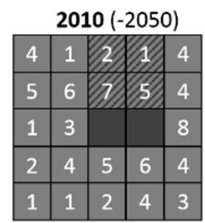

$2010(-2030)$

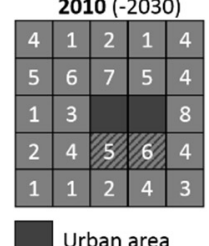

Urban area

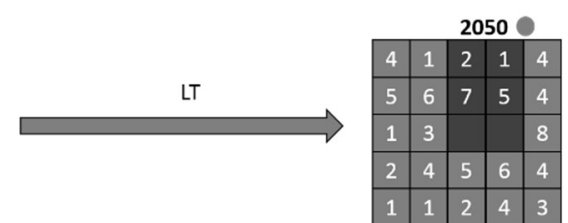

2050 .

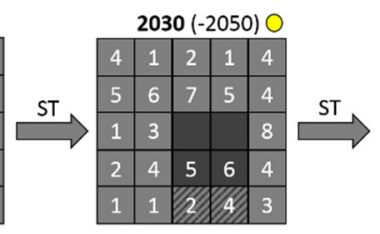
3 Agricultural area

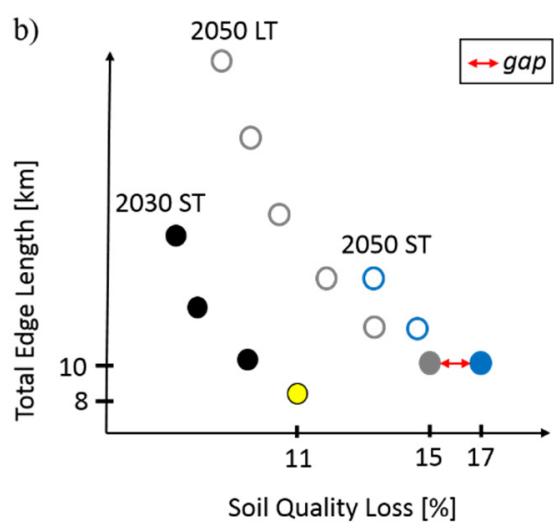

Figure 1. a),b): Toy experiment. In 2010 there are two urban cells and 23 agricultural cells. We aim at finding the most compact solution in 2050 with the lowest loss in soil quality (numbers in cells represent soil quality). This corresponds to a solution at the bottom-right of a front of non-dominated solutions. For example, the Total Edge Length for the pattern marked with the grey dot would be 10 (including the two edges at the upper end of the pattern). The loss of soil quality would be $2+1+7+5=15$. To exemplify long-term planning (LT), we designate four agricultural cells to future urban areas (zoning area in the upper left pattern). By 2050, these zoned areas are becoming urban (upper right pattern). In the shortterm (ST) planning case, we first only designate 2 cells to future urban use. The most compact solution with the lowest loss in soil quality causes a loss of 11 soil points and a Total Edge Length of 8 (lower left pattern). By 2030, the designated zones are converted into urban and 2 new cells are designated for urban areas (lower central pattern). By 2050, these two zones turn into urban, which causes a Total Edge Length of 10 and a Soil Quality Loss of 17 since 2010. 


\subsection{Real-world application}

In contrast to the toy problem situation above, we found that short-term planning didn't result in non-optimal solutions when optimizing the urban pattern in the municipality of Uster in Switzerland. First, there was no gap between the fronts produced in the optimization process simulating long-term planning and the fronts produced in the optimization process simulating short-term planning (Figure 2). Second, the most compact patterns resulting from the simulations until 2050 (adding to land-use map in 2010 two times 106 new urban cells (short-term planning) and adding 212 new urban cells (long-term planning) are very similar and both contain the pattern found when simulating short-term planning until 2030 (Figure 3).

The fact that short-term planning didn't result in non-optimal solutions means that urban planners could be recommended to develop a zoning plan for 2030 first and afterwards to create a second plan for 2050. If they would instead do the zoning for 2050 (starting in 2010), they might designate too many agricultural cells to future urban areas (oversized zones) because the demand for urban areas may have been overestimated. However, urban planners will try to avoid oversized zones because they often result in unforeseen and nonoptimal urban patterns, as the actual allocation of urban areas within zones is strongly driven by economic factors and does usually not account for externalities (Holcombe and Williams, 2012).

The simulation of the short-term planning reveals that a chosen preference, expressed as the weight for the two objectives, strongly determines which solution we are able to reach in the long run (Figure 2 a, Figure 4 a). Selecting a preference early (Figure 2 a) will allow us to reach a wider range of solutions than when selecting the preference in a later stage (Figure 4 a). This could mean that if there is uncertainty about the right preferences, short-term planning may be more reasonable, as an early adjustment in preferences may have a larger effect than later adjustments.

Interestingly, we found that two patterns having almost the same objective values showed quite some differences in the decision space (i.e., in the land-use patterns). While most of the 212 urban cells allocated in the two optimization runs (short- and long-term simulation) were at the same location, more than 30 of them were found at different locations (comparison of pattern 441 and 336). Instead of preserving the diversity of solutions within in the objective space, accomplished by the so-called crowding distance in NSGA-II (Deb et al., 2002), it may be helpful to also preserve diversity in the decision space. Taking such diversity into account may not only be helpful to get as close as possible to the true Pareto-Front, but may also be used when trying to preserve non-dominant solutions that are close to the Pareto Front in the objective space, but very different in the decision space. This can be very useful, when decision makers want to evaluate a wide range of options (e.g. Kwakkel et al., 2016).
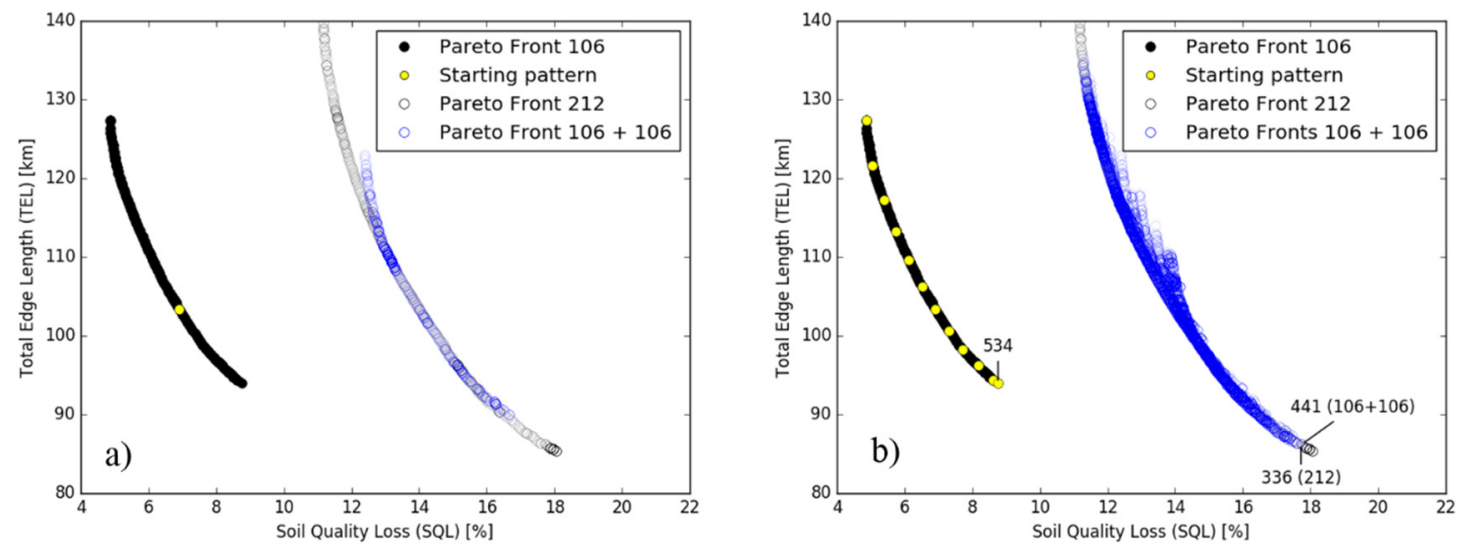

Figure 2. a),b): a) The figure shows 3 non-dominated fronts. Two of them represent the short term-planning process (black dots with fill, blue dots without fill color) while one of them represents the long-term planning (grey dots without fill color). Short term planning involved one optimization run placing 106 new urban areas (which corresponds approximately to the year 2030) onto the current land-use map (from 2010). After that, one pattern from the front of non-dominated solutions was selected (yellow dot) and again 106 new urban areas were placed onto this land-use pattern. In total there were 212 new urban areas. For the long-term planning process, 212 new urban areas were allocated in one single optimization run. b) In total the figure shows 14 non-dominated fronts. Each yellow dot represents a land-use pattern that was used as a starting point for an optimization run (short-term planning process). Each blue front is a result from the optimization process based on one of the starting patterns. The front produced for long-term planning until 2050 is largely invisible as it is mostly covered by the 12 blue fronts. The most compact pattern in the year 2030 (pattern 534), the most compact one in 2050 for short-term planning (pattern 441) and the most compact pattern of long-term planning (pattern 336) are shown in Figure 3. 

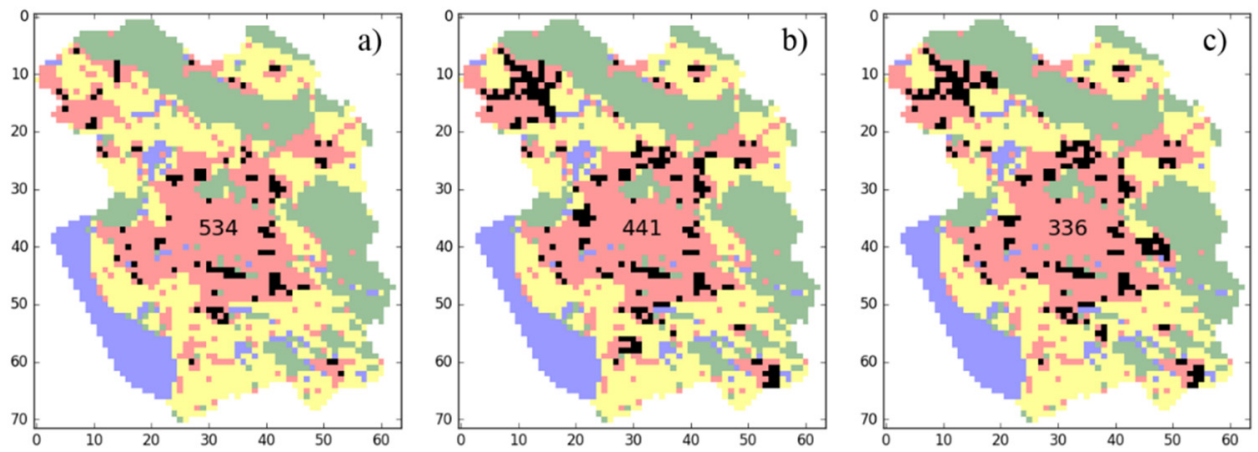

Figure 3. a)-c): Red cells: Urban areas; Yellow cells: Agricultural areas; Green Cells: Forest areas, Blue Cells: Water; Black Cells: New Urban areas which were allocated in the optimization process. a) Pattern 534. Most compact pattern when 106 new urban areas are added in the optimization process (short-term planning) to the current land-use in the year 2010. b) Pattern 441. Most compact pattern when 106 new urban cells are added to pattern 534. c) Pattern 336. Most compact pattern, when 212 new urban cells are added to the current land-use in the year 2010.
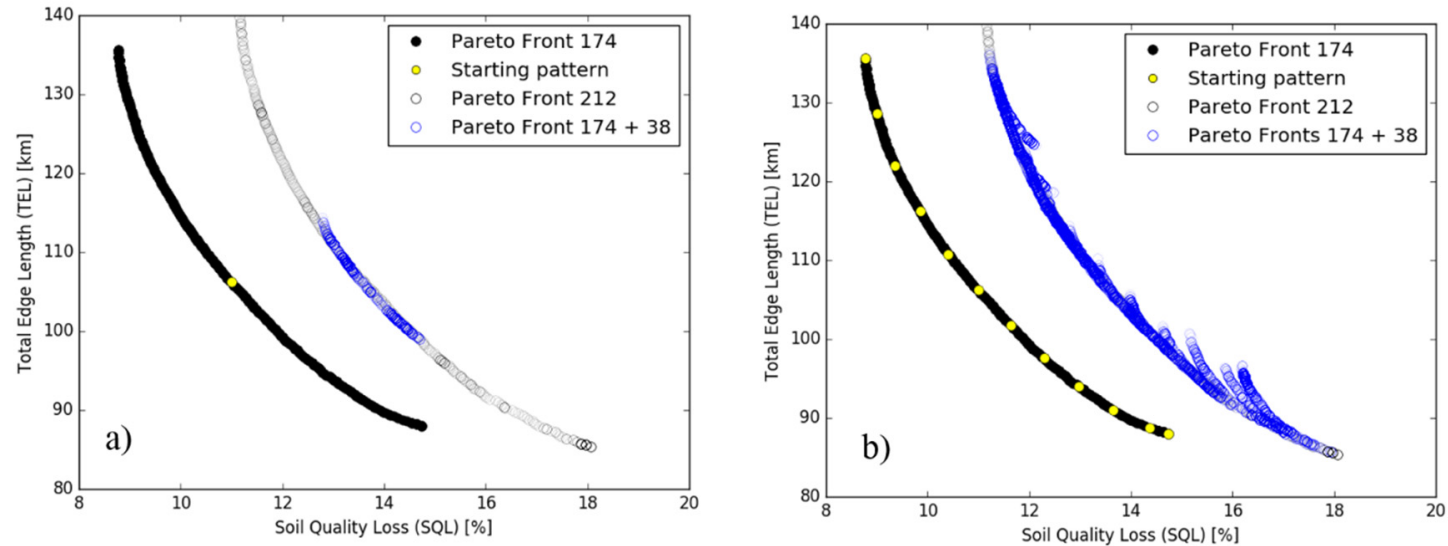

Figure 4. a), b): a) The figure shows 3 non-dominated fronts. Two of them represent the planning process when first using a conservative demand estimate of 174 ha (black dots with fill) and afterwards adding 38 ha more of urban areas (blue dots without fill color). This could also be interpreted as a short-term planning process (2010-2043-2050). The third front was produced for the median demand prediction and corresponds to the long-term planning until 2050 (grey dots without fill). b) In total the figure shows 14 non-dominated fronts. Each yellow dot represents a land-use pattern that was used as a starting point for an optimization run (conservative scenario/short-term planning process). Each blue front is a result from the optimization process based on one of the starting patterns. The front produced for the median demand estimate (or longterm planning until 2050) is largely invisible as it is covered by the blue fronts.

\subsection{Comparison of toy experiment and real-world application}

The toy experiment showed that a short-term planning process can be problematic if it prevents finding an optimal solution on the longer run. However, the real-world application showed that this doesn't necessarily have to be the case. It will depend on the spatial pattern of the current land-use and the spatial pattern of the soil quality, whether a short- or a long-term planning process is recommendable. In order to be able to give a more generalizable recommendation to urban planners, it would thus be necessary to apply our analysis to a large variety of different municipalities.

Building on this work, future analysis could address the question whether it is possible to identify a minimum length of the planning periods with which it would still be possible to find the best solutions in comparison to a long-term planning period (i.e., for the longer run). In addition, it could be necessary to formulate the whole problem in a more dynamic approach (Zhiqiong and Bojin, 2010), as urban planners may not only want to identify the best possible solutions at a defined point in time but also find good solutions applicable over a period of time.

\section{CONCLUSIONS}

In this paper, we have shown that multi-objective optimization can be used to support urban planners in choosing the right planning horizon. The presented methodological approach and results show that multi- 
objective optimization can be more useful than just providing decision-makers with optimal solutions for complex problems. We believe that demonstrating innovative approaches of how to use multi-objective optimization has the potential to enhance decision-making processes and will foster the application of multiobjective optimization in the area of urban modelling and planning.

In a concrete example using multi-objective optimization we showed that short-term planning would be recommended for the urban planning problem in the municipality of Uster in Switzerland. However, it depends on the spatial pattern of the current land-use and the spatial distribution of the soil quality, whether a short- or a long-term planning process is recommendable. Building on this work, future analysis could address the question whether it is possible to identify a minimum length of the planning periods with which it would still be possible to find the best solutions in comparison to a long-term planning period. The methodology we developed could help planners to identify the right planning horizons for a large variety of spatial planning problems.

\section{ACKNOWLEDGMENTS}

Funding for this work was provided by the Swiss National Science Foundation (SNSF). It was part of a Doc.Mobility grant (P1EZP2_162222) and the project SUMSOR (406840_143057), which is part of the National Research Programme "NRP 68 - Sustainable use of soil as a resource". This material is also based in part upon work supported by the U. S. National Science Foundation under Cooperative Agreement No. DBI0939454. Any opinions, findings, and conclusions or recommendations expressed in this material are those of the authors and do not necessarily reflect the views of the National Science Foundations.

\section{REFERENCES}

Aerts, J., Eisinger, E., Heuvelink, G. B. M. \& Stewart, T. J. (2003). Using linear integer programming for multi-site landuse allocation. Geographical Analysis, 35, 148-169.

Artmann, M. (2015). Managing urban soil sealing in Munich and Leipzig (Germany)-From a wicked problem to clumsy solutions. Land Use Policy, 46, 21-37.

Caparros-Midwood, D., Barr, S. \& Dawson, R. (2015). Optimised spatial planning to meet long term urban sustainability objectives. Computers Environment and Urban Systems, 54, 154-164.

Deb, K., Pratap, A., Agarwal, S., \& Meyarivan, T. (2002). A fast and elitist multiobjective genetic algorithm: NSGA-II. Ieee Transactions on Evolutionary Computation, 6, 182-197.

FAO and ITPS (2015). Status of the World's Soil Resources (SWSR) - Main Report. Food and Agriculture Organization of the United Nations and Intergovernmental Technical Panel on Soils, Rome, Italy

Fortin, F. A., De Rainville, F. M., Gardner, M. A., Parizeau, M., \& Gagne, C. (2012). DEAP: Evolutionary Algorithms Made Easy. Journal of Machine Learning Research, 13, 2171-2175.

Haque, A. \& Asami, Y. (2014). Optimizing urban land use allocation for planners and real estate developers. Computers Environment and Urban Systems, 46, 57-69.

Holcombe, R. G., \& Williams, D. W. (2012). Urban Sprawl and Transportation Externalities. 2012, 40, 16.

Humbel 2009. Arealstatistik nach Nomenklatur 2004 - Standard. Geostat Datenbeschreibung. BFS Bundesamt für Statistik (eds.). Bern.

Kwakkel, J. H., Walker, W. E., \& Haasnoot, M. (2016). Coping with the Wickedness of Public Policy Problems: Approaches for Decision Making under Deep Uncertainty. Journal of Water Resources Planning and Management, 142, 5.

Malczewski, J. (2015). Multicriteria decision analysis in geographic information science, New York: Springer.

McGarigal, K., Cushman, S., \& Ene, E. (2012). FRAGSTATS v4: Spatial Pattern Analysis Program for Categorical and Continuous Maps. Computer software program produced by the authors at the University of Massachusetts, Amherst.

Rittel, H. W. J., \& Webber, M. M. (1973). Dilemmas in a general theory of planning. Policy Sciences, 4, 155-169.

Ryerkerk, M., Averill, R., Deb, K., \& Goodman, E. (2012). Meaningful representation and recombination of variable length genomes. Proceedings of the 14th annual conference companion on Genetic and evolutionary computation. Philadelphia, Pennsylvania, USA: ACM.

Schwaab, J., Deb, K., Goodman, E., Lautenbach, S., Van Strien, M., \& Grêt-Regamey, A. (2017). Reducing the loss of agricultural productivity due to compact urban development in municipalities of Switzerland. Computers, Environment and Urban Systems, 65, 162-177.

Stewart, T. J., Janssen, R., \& van Herwijnen, M. (2004). A genetic algorithm approach to multiobjective land use planning. Computers \& Operations Research, 31, 2293-2313.

Walker, W. E., Haasnoot, M. \& Kwakkel, J. H. (2013). Adapt or Perish: A Review of Planning Approaches for Adaptation under Deep Uncertainty. Sustainability, 5, 955-979.

Zhiqiong, B., \& Bojin, Z. (2010). Perspectives in Dynamic Optimization Evolutionary Algorithm. Advances in Computation and Intelligence. Proceedings 5th International Symposium, ISICA 2010, 338-348.

Zitzler, E. (1999). Evolutionary algorithms for multiobjective optimization: methods and applications. Ph.D. thesis. RWTH Aachen. Aachen: Shaker. 